\title{
Tensiones y adecuaciones de los trabajadores en el marco de la ejecución de la política social chilena
} Tensions and adjustments of workers in the context of the implementation of Chilean social policy

\author{
Javiera Beatriz Pavez Mena \\ Pontificia Universidad Católica de Valparaíso
}

\begin{abstract}
Resumen
En Chile, las políticas sociales de infancia son implementadas por organizaciones del tercer sector. En este estudio propuse identificar las tensiones y adecuaciones de los trabajadores y las trabajadoras que implementan programas de infancia. Trabajé desde la perspectiva de la etnografía crítica de la acción pública, considerada como una estrategia de develamiento de los vínculos de dominación que estructuran la ejecución de la política social. Realicé observaciones participantes y entrevistas en profundidad a trabajadores ejecutores de las políticas, levantando como principal resultado tensiones asociadas a la resignación de los y las trabajadoras, y a una vivencia de temporalidades disímiles; y, como principales adecuaciones, el trabajo colaborativo y la habilitación. Así, las prácticas de los trabajadores del programa social de infancia nos convocan a una reflexión en torno a cómo las condiciones de ejecución favorecen determinadas construcciones de sujeto en el marco de la acción social del Estado neoliberal.
\end{abstract}

Palabras clave: Políticas Públicas; Etnografía Crítica; Trabajadores Sociales; Nueva Gestión Pública

\begin{abstract}
In Chile, children's policies are developed by third sector organizations. In this study, I proposed to identify the tensions and adjustments of the workers implementing a child welfare programme. I worked from the perspective of the critical ethnography of public action, considered as a strategy to reveal the links of domination that structure the execution of social policy. I made participant observations and interviews with policy workers, raising as a main result tensions associated with the resignation of workers, and an experience of dissimilar temporalities, and as main adjustments collaborative work and qualification. Thus, the practices of the workers of the social childhood program call for reflection on how the conditions of execution produce certain constructions of subjectivity within the framework of the social action of the neoliberal State.
\end{abstract}

Keywords: Public Policy; Critical Ethnography; Social Workers; New Public Management 


\section{INTRODUCCIÓN}

En Chile, las trasformaciones del Estado derivadas de la aplicación de las políticas del llamado "Consenso de Washington" modificaron los vínculos establecidos entre el Estado, el mercado y la sociedad civil, repercutiendo en un cambio en la orientación de las políticas públicas y, en especial, de las políticas sociales (Barros Aguirre, 2006; Ramírez, 2009;). Se instaló la nueva gestión pública (NGP) que buscó transformar la cultura de las organizaciones, introduciendo a privados en el desarrollo de la acción pública (Fardella et al., 2016; Bezes et al., 2012; Hood, 1991).

Particularmente, en el campo de las políticas sociales de infancia chilenas, la incorporación de privados propia de la NGP se expresa en que los programas sociales son fundamentalmente ejecutados por organizaciones del tercer sector, tales como Organizaciones No Gubernamentales (ONG), Fundaciones y Corporaciones. Estas organizaciones postulan a licitaciones de programas que abren los departamentos estatales, llevándose a cabo procesos de selección de las propuestas presentadas y celebrándose, posterior a ello, un convenio, acordándose las condiciones del traspaso de fondos públicos para la ejecución de los programas adjudicados (Pavez et al., 2019).

En este marco, el Estado se limita a la regulación y supervisión eficiente de los gastos, privilegiando los resultados sobre los procesos con la ayuda de mecanismos de rendición de cuentas (Fardella et al., 2016). Así, en el caso de las políticas sociales de infancia en Chile, el rol de supervisión y fiscalización se le atribuye al Servicio Nacional de Menores (Ley N²0.032).

De este modo, las ONG aumentan su acción directa en cuanto a la ejecución de las políticas sociales de infancia dentro de la sociedad, aun cuando se debilitan y subordinan, transformándose en entidades de prestación de servicios que, mediante licitaciones, compiten para adjudicar el financiamiento que permite el funcionamiento de los programas (Sánchez y Villarroel, 2017).

Dada esta estrategia de licitación por medio de la cual se desarrolla la tercerización de los programas, las acciones propias de éstos se realizan en contexto de escasez de recursos y precariedad en las condiciones contractuales. La mayoría de los programas se conforman por trabajadores con contratos a honorarios y con falta de continuidad por ser dependientes de la renovación del convenio resultante de la licitación (Haz et al., 2012).

Así, el trabajador y trabajadora del área social debe instalar o asegurar, por medio de la ejecución de programas, un efecto redistributivo o de rescate en aquellas familias más vulnerables del país desde una condición de precariedad laboral (Dávila, 1998; Torres, 2009). 
Considerando el carácter activo y colectivo del ejercicio de los derechos, las tensiones que pueden estar generándose al interior de los programas sociales a partir de este contexto precario pueden influir en el adecuado cumplimiento de los objetivos que éstos se proponen.

En el marco de esta problemática, por medio del presente estudio inserto en una investigación mayor, propuse identificar las tensiones y adecuaciones de los trabajadores y las trabajadoras de un programa social de infancia que se ejecuta en condiciones de precariedad. Realicé esta identificación desde la perspectiva teórico-metodológica de la etnografía crítica de la acción pública, considerada como una estrategia de develamiento de los vínculos de dominación que estructuran la ejecución de la política social (Dubois, 2012). Desde este enfoque, se propone que las acciones del Estado no serían definitivas, sino que -como señala Michel Foucault (2004/2007) - serían efecto de constantes estatizaciones, transacciones y tensiones que modifican la autoridad central. Se distinguen tensiones y adecuaciones que atravesarían el quehacer de los y las trabajadores y, a partir de una matriz de análisis foucaultiana, se desarrollan conclusiones en torno a cómo las condiciones de ejecución favorecen determinadas construcciones de sujeto en el marco de la acción social del Estado neoliberal (Foucault, 1982; 2004/2007; Lorenzini, 2018; Paltrinieri, 2013).

\section{ETNOGRAFÍA CRÍTICA DE LA ACCIÓN PÚBLICA}

La perspectiva de la etnografía crítica de la acción pública considera que las políticas existen a partir de lo que desarrollan quienes las implementan. De esta manera para su abordaje se privilegia la observación de las interacciones dado que estas permitirían conocer y comprender el funcionamiento del Estado social contemporáneo (Dubois, 2012).

Desde este enfoque, la acción pública enmarcada en el Estado deviene y no se constituye en un marco inamovible previamente dado e inalterado en su despliegue. El Estado se comprende así como un cúmulo de aspectos materiales e ideológicos (Sharma y Gupta, 2006), como efecto de transacciones incesantes que modifican y desplazan las relaciones de poder (Foucault, 2004/2007). Esta repetición banal de las prácticas cotidianas reproduce el Estado a través del tiempo y el espacio, reproduciendo explotaciones y desigualdad, y conteniendo al mismo tiempo la posibilidad de la resistencia (Dubois, 2012; Sharma y Gupta, 2006).

El abordaje etnográfico crítico de la acción pública, y particularmente la observación directa de las interacciones, constituye una elección de método que permite visualizar las prácticas de control que permiten aprehender las ten- 
dencias de las políticas sociales contemporáneas. Asimismo, es una herramienta que permite visualizar las contradicciones de la política, el control prometido en nombre del rigor jurídico al mismo tiempo que la incertidumbre de los criterios (Dubois, 2012; Lipsky, 1980).

Dicho abordaje nos permite poner especial atención en cómo la gente percibe al Estado, cómo se forman esos entendimientos y cómo el Estado se manifiesta en la vida de las personas (Sharma y Gupta, 2006). Se problematiza de este modo el vínculo entre el Estado y la sociedad, sociedad civil o la comunidad, reconceptualizando las definiciones y las fronteras entre lo público y lo privado.

En el ámbito de las políticas sociales, se han desarrollado estudios que permiten conocer qué nociones constituyen las prácticas estatales en cuanto a los ciudadanos receptores - denominados usuarios o beneficiarios- y a los ciudadanos ejecutores - los trabajadores de la política social-, recogiendo una perspectiva interaccional (Coutant y Eideliman, 2013; De La Maza, 2012; Dubois, 2015/2020; Fassin, 2010; Wanderley, 2009).

Vincent Dubois (2015/2020), a partir de la etnografía desarrollada en las ventanillas de atención a usuarios precarios, constata la construcción de un doble cuerpo en los trabajadores, quienes por un lado encarnan al Estado y por otro, se mantienen como personas generando roles de contención. De este modo, se ejerce simultáneamente un lazo social y coerción, constatándose también la interpelación de los usuarios hacia este doble cuerpo. Según el autor, este análisis micro-social permite abrir una visión global de los lazos sociales y la acción pública, revelando los límites de las políticas sociales en Francia.

Por su parte, Didier Fassin (2010), a partir de los estudios en políticas de abordaje del $\mathrm{VIH}$, constata la construcción de dos comunidades, una defendiendo el derecho a la vida y la otra el derecho a la justicia. A partir de su estudio etnográfico, Fassin (2010) constata cómo aparecen objetos clásicos de las políticas públicas, planteados desde el punto de vista de los derechos económicos y sociales, y sobre una idea de justicia social determinada. El autor discute cómo en las políticas sociales se ha construido una biolegitimidad, viéndose desplazado el principio de justicia social aplicado a la asignación de recursos públicos por un principio de justicia social que da a los que piden, articulándose así una economía moral contemporánea.

En cuanto a las políticas sociales de infancia con infractores de ley, Isabelle Coutant y Jean-Sébastien Eideliman (2013) se preguntan por las condiciones bajo las cuales las interacciones entre adolescentes, familias y trabajadores en los Centro de Adolescentes participan en un proceso de control social o, por el 
contrario, permiten formas de empoderamiento para los adolescentes que adscriben. Para ellos, en estas formas contemporáneas de intervención no hay sólo una cuestión de control social y de dominación institucional en la consejería a adolescentes, pues los trabajadores involucrados no desarrollan sus prácticas desvinculados de su reflexividad y distancia crítica. Los autores sostienen que las transformaciones macrosociales informan la experiencia moral de los actores con la escena local, de acuerdo a sus membrecías y sus posiciones sociales, generándose un proceso de redistribución de capital simbólico.

En Bolivia, Fernanda Wanderley (2009), a partir de la consideración que las interacciones cara a cara son una instancia de formación de las nociones y vivencias de los derechos ciudadanos, desarrolla una etnografía en los servicios de registro e identificación. Cuando las interacciones ocurren en las oficinas públicas, la calidad del trato que los ciudadanos reciben de los funcionarios es una medida de equidad de los mismos en la sociedad y el Estado. Concluye que las identidades ciudadanas reforzadas en estos encuentros son las colectivas en detrimento de la ciudadanía individual.

Finalmente, Francisca De La Maza (2012) en la Araucanía desarrolla una aproximación desde la etnografía de Estado a la implementación de la Ley Indígena 19 253, sosteniendo que dicho abordaje permite observar el Estado desde adentro. Poniendo el foco en las oficinas de atención a la población indígena, observa las interacciones entre los agentes estatales y los usuarios, relevando cómo -en la puesta en ejecución de esta Ley- las prácticas sociales vinculadas a su implementación pasan a formar parte de las prácticas del Estado. Para De La Maza (2012), la oficina constituye un espacio de implementación de la política y de construcción del Estado en lo local. Además, constituye un lugar de resistencia política, al abrir nuevas formas de relación y de visibilización de los indígenas en lo local.

De esta manera, la investigación etnográfica prolongada, asociada a la presencia continua con los interventores y la observación de sus prácticas, permite comprender el funcionamiento del Estado social contemporáneo, así como sus dinámicas situadas de construcción y transformación (Dubois, 2012).

Esta perspectiva llevada al análisis del Estado Latinoamericano, permitiría la visibilización de su construcción tensionada desde las situaciones de desigualdad que han puesto en evidencia la incapacidad que ha tenido la instalada economía de libre mercado para la superación de las antiguas y nuevas problemáticas de nuestras sociedades (Álvarez y Sáenz, 2014). Expresado desde la política social de infancia, visibilizar cómo la precariedad presente en la ejecución de sus programas influye en la construcción del Estado que desde allí se realiza. 
En esta investigación, consideré la etnografía crítica como un proceso por medio del cual es posible construir conocimiento de una situación problemática para la sociedad con miras a su transformación. De este modo, en esta investigación no me sitúo desde una posición neutral, sino que me comprometo desde una visión crítica de la ejecución de las políticas sociales de infancia y me propongo aportar conocimientos sistemáticos para su transformación (Street, 2003). Como investigadora me sitúo como agente, llevando a cabo una etnografía en la que se desarrollaron conversaciones políticamente sensibles que problematizan la propia situación de los trabajadores (Fine, 1994).

\section{MÉTODO}

De esta manera, me propuse analizar las prácticas de los trabajadores y trabajadoras de un programa social de infancia ejecutado en un contexto de precariedad laboral. Para ello, accedí a cómo se ejecuta la política social de infancia desde la observación participante de la cotidianeidad de un programa social, desarrollando una observación que consideró la descripción del entorno, la descripción de los participantes, la descripción del entorno físico y material, la descripción de las interacciones y el registro de las conversaciones e interacciones. Aun cuando en el presente estudio se privilegiará la producción en torno al hacer o al saber hacer de los y las participantes.

Desde una aproximación postestructuralista, trabajé desde un enfoque cualitativo, que permitió situarme desde el punto de vista de quienes participan de la investigación, llevando la atención a los procesos, patrones significativos y características estructurales, contribuyendo a una mejor comprensión de la realidad social (Flick et al., 2004; Núñez et al., 2014).

La unidad de análisis fue un programa social de infancia denominado Programa de Intervención Especializada (PIE). Éste constituye una de las variadas líneas programáticas del SENAME, el Servicio Nacional de Menores, que está abocado a administrar y tercerizar parte de la acción social del Estado chileno en materia de infancia. El programa estudiado tiene como objetivo contribuir a la reparación del daño ocasionado por negligencia grave, abandono y explotación. Se propone interrumpir la sintomatología y/o de las conductas que transgreden derechos de otras personas.

El PIE estudiado se encuentra ubicado en la región de Valparaíso, a $120 \mathrm{~km}$ de Santiago, la capital de Chile, y atiende a un total de 60 niños y niñas y sus familias. A junio de 2020, existían poco más de 100 PIE ejecutados por diversas ONG u otros organismos colaboradores de SENAME. 
Trabajé con los trabajadores y trabajadoras que ejecutaban el programa, quienes voluntariamente accedieron a participaron de la investigación y facilitaron el acceso a sus diversos espacios de trabajo tales como las dependencias del programa, reuniones de coordinación con la red educativa y espacios informales de intercambio.

El diseño etnográfico me permitió formar parte de la cotidianeidad del programa seleccionado - desde el punto de vista de los participantes - durante un período de 4 meses (Íñiguez, 1999; Núñez et al., 2014; Silva y Burgos, 2011). A partir este diseño fue posible profundizar en eventos o situaciones sociales específicas, realizando un trabajo de campo no extenso (Jeffrey y Troman, 2004).

Registré el proceso etnográfico en un diario de campo, realizando anotaciones por cada visita al centro o contacto realizado. Además, desarrollé 47 entrevistas con 19 personas, organizadas en 10 entrevistas en profundidad, las que fueron transcritas siguiendo el método Jefferson y 37 entrevistas conversacionales registradas en el cuaderno de campo. Posterior a ello, codifiqué la totalidad del material producido siguiendo una lógica deductiva, a partir de los ejes conceptuales de la investigación, y luego inductiva, considerando lo referido por los y las participantes. Desarrollé un análisis de contenido, agrupando por similitud y reduciendo a 31 códigos que fueron registrados en un libro de códigos. Posterior a ello, identifiqué patrones que luego ensamblé considerando mi pregunta de investigación. De esta manera, constituí estructuras conceptuales que informan respecto de los actos de los y las trabajadoras, estableciéndose conclusiones a partir de hechos pequeños, de contextura densa (Geertz, 2005). Estos se presentan como prácticas de tensiones y adecuaciones en el siguiente apartado.

Finalmente, en el desarrollo del estudio -que se enmarca en una tesis doctoral debidamente visada en cuanto a sus lineamientos éticos - consideré el resguardo a la intimidad de los participantes (en el presente artículo se utilizan pseudónimos) y mantuve la confidencialidad de los datos producidos. Ello como resultado de un proceso de construcción de un consentimiento que implicó diálogos y presentaciones en diversas instancias con distintos actores de la organización ejecutante y el programa. De este modo, durante el proceso fue conocido el propósito de la investigación por parte de todos los actores, quienes además decidieron en consenso participar de esta investigación.

\section{RESULTADOS}

A continuación, se presentan las estructuras conceptuales emergidas del análisis, organizadas en tensiones y adecuaciones realizadas por los trabajadores y 
trabajadoras del programa social. Se acompañan de las citas y extractos del cuaderno de campo más representativos.

\section{Tensiones del trabajo precario}

\section{La Resignación}

La resignación la comprendemos como aquellas prácticas en las que los y las trabajadoras dan cuenta de estar en aceptación de su condición laboral precaria. De maneras diversas, muestran una cierta conformidad con las situaciones en las que se ven envueltos en su cotidianeidad de ejecución del programa.

Hay numerosas expresiones que entendemos como muestras de resignación: "para que calentarse la cabeza", "no nos hacemos mala sangre", "uno sabe cómo funciona esto". Como señala un trabajador:

236. E2: =por eso yo te comentaba >como que trato de no estresarme tanto < porque yo sé en lo que estoy >yo sabía a lo que venía en cierto modo< sabía dónde me estaba metiendo ¿cachai?=. (Daniel, entrevista personal, marzo del 2016)

Cabe destacar que esta resignación precede a una profunda frustración que implica no poder ejecutar las acciones del programa en las condiciones que son las que consideran más pertinentes para hacerlo. Ello sumado a la frustración por no poder desarrollarse profesionalmente y de acuerdo a su formación, de no poder desarrollar su vida como esperarían.

Esta frustración es denominada por algunos como "rabia contra la estructura", "rabia contra el sistema", reconociendo que en último término hay un sistema de ejecución de la política que precariza.

144.E2: [yo (x) yo creo que] ese:: es un:: (x) un::: (x) un arcoiris de hartos colores eh::: >a propósito de que < yo creo que el::: (.) (.hhh) justamente

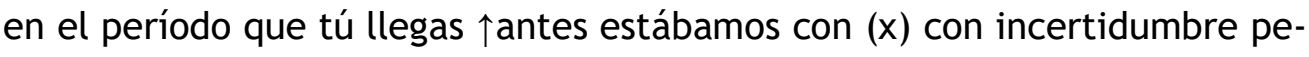
ro manteniendo como::::: el sistema iya? >Luego tenemos estas otras< certezas que se basan en::: $(\mathrm{x})$ en:: $(\mathrm{x})$ en incertidumbres ¿cierto? Certezas de que si nos va bien, no necesariamente vamos a estar todos eh:: >0 sea no vamos a estar todos< de hecho (.hhh) como certeza, no depende de los resultados porque eso no lo sabemos (.hhh) $Y$ ahí también hay:: como::: con::: no sé $(\mathrm{x})$ no sé si contagiado o yo contagiando a los chiquillos con $(\mathrm{x})$ con esta cosa (.) como medio cabizbaja:::: (.hhh) con:: finalmente con (.) una cuestión $\downarrow$ desilusionante:: pero no $(x)$ no:: también le $(x)$ le di una vuelta y no con $(\mathrm{x})$ con la institución propiamente tal sino con el sistema. Un sistema que no (x) no te::: (.) pese a tener buenos resultados, pese a tener avances con los chiquil:: llos y no estar exentos a un montón de dificultades, pero como:: > poniéndole dándole< a la pega (.hhh) aun así como 
que no existe como un::: como cuida:::do o:: (x) o condiciones que aseguren eh:: el::: (x) el bienestar no solamente de estas familias entre comillas que están en una situación compleja >sino también< (.hhh) de las familias de mis compa::ñeros, de mi fami::lia, de las personas con las que conformamos el equipo (.hhh) y $\uparrow$ ahora:: (.hhh) estoy en otra::: cambió el switch, porque:: también SIENTO, yo al menos, la responsabilidad de eh::: esta cuestión hay que mantenerla hasta que::: sea. (Bernardo, entrevista personal, marzo de 2016)

De este modo, entendemos el término sistema como aquel ordenamiento propio de las políticas sociales de infancia que implica el funcionamiento sujeto a licitaciones y adjudicaciones de proyectos que no aseguran condiciones para el bienestar de los trabajadores y sus familias, y de los niños, niñas y sus familias que participan del programa.

Este sistema genera rabia en los trabajadores y trabajadoras, frustración que es verbalizada en el cotidiano del programa. Sin embargo, en sus expresiones y acciones cotidianas es posible identificar que la frustración en algunos da paso a la resignación.

Desde el punto de vista de los Estados de Ánimo (Ascorra, 2009) comprenderemos la resignación como "aquella emocionalidad que tiende a paralizar la acción orientada a las transformaciones" (p. 22). La resignación en los y las trabajadoras del programa implica una normalización de la situación precaria que viven y una consideración de que el trabajo es así, es decir, que las condiciones precarias son transversales a todos sus potenciales ámbitos de desarrollo laboral.

La resignación los sitúa en un estado de ánimo desde el cual opera una despotenciación (Ascorra, 2009), impidiendo la generación de resistencias activas y colectivas. Es decir, esta resignación se presenta en relación a las condiciones de ejecución de los programas, es decir, a la constatación de que así opera la política social de infancia y que no tiene sentido esperar algo distinto.

\section{La Temporalidad}

Es posible identificar una segunda tensión vinculada a la dimensión de la temporalidad. Los trabajadores y trabajadoras distinguen un tiempo lento propio de la relación que establecen con los usuarios y un tiempo rápido propio de las gestiones con las instituciones y de la rendición de cuentas que deben realizar a otras organizaciones externas.

Representando la vivencia de los trabajadores, uno de los trabajadores retrata esta situación haciendo una analogía con las fotografías de un primer plano fijo y un fondo en movimiento: 
181. E1: sí po, es (x) es curioso porque es como:: $\mathrm{m}:::$ : (.) es como (x) es un poco $>$ como si se detuviera el tiempo<=

182. E2: $=M::=$

183. E1: =ंcachai?=

184. E2: $=[$ sí $]$

185. E1: [es] como::: que::: es como no sé [es]

186. E2: [es] como estas películas que $(\mathrm{x})$ que $>$ no sé si tú has visto< que es como que se mueve lento pero se $(x)$ la $(x)>$ los autos que pasan rápido por la carretera $<=$

187. E1: =sí=

188. E2: =no sé [si me logras]

189. E1: [>sí $(x)$ sí $(x)$ sí $(x)$ sí<]=

190. E2: =entender=

191. E1: $=$ sí=

192. E2: = ¿te fijas? Entonces >dada la ocurrencia de los ca::sos, la contingencia y todo lo demás< pero nosotros estamos como::=

193. $\mathrm{E} 1:=$ como en un tiempo lento=

194. E2: $=$ claro=

195. E1: =como que estuvieran en dos [temporalidades]

196. E2: [a destiempo]=

197. E1: =por decir=

198. E2: $=[\mathrm{sí}]$.

(Bernardo, comunicación personal, marzo de 2016)

Cabe destacar que la construcción del vínculo está situada en un tiempo lento, que se ve constantemente amenazado por el énfasis en los indicadores de resultados y en la estadística que deben entregar a fin de mes, las cuales estarían situadas en un tiempo rápido.

Ambos tiempos son posibles de identificar en las prácticas de los trabajadores. Las temporalidades varían en distintos momentos del mes, asociados a los hitos de rendición de cuentas.

"Es fin de mes me dicen al verme entrar". Los últimos y fundamentalmente el último día hábil del mes es el más activo porque [los trabajadores y trabajadoras] deben dejar completadas las fichas, registros e intervenciones en el sistema computacional. Se los ve escribir apurados en las carpetas y turnarse los computadores para alcanzar a cerrar los registros antes de la hora del fin de la jornada. (Entrada de diario de campo, mayo de 2016)

Desde la perspectiva del análisis de las temporalidades de la acción del care, resulta relevante este elemento del tiempo lento y el tiempo rápido. Como señala Marc Bessin (2014) habría un tiempo de Cronos, expresado en años, horas, segundos, cuyo uso se ha generalizado para convertirse en hegemónico con el desarrollo del capitalismo. Este tiempo cronológico sería la medida cuantitativa 
que se impone como argumento principal en nuestras sociedad, entregando un marco universalizante a las actividades. Por otra parte, habría un tiempo de Kairos, que se vincula a aquello que no puede ser programado en la acción de estar con otros y que se relaciona con la capacidad de aprehender las situaciones a medida que se desarrollan, en todas sus dimensiones cualitativas.

De este modo, siguiendo a Bessin (2014), podríamos señalar que el tiempo lento de los trabajadores obedece al tiempo de Kairos, a un tiempo del vínculo con el niño, niña o adulto, el buen momento para intervenir que no puede ser programado con anticipación.

El tiempo rápido por su parte, correspondería al tiempo de Cronos y respondería a todas aquellas acciones que el equipo debe hacer en términos de responder a los tiempos de organizaciones externas de justicia y de registro de SENAME.

Esta duplicidad de tiempo es vivida con desconcierto por los y las trabajadoras y es generadora de una tensión que no se expresa con fluidez, pero que, sin embargo, es visible desde sus prácticas.

Mauricio Sánchez y Rosa Villarroel (2017), a partir de un análisis de las tensiones en la intervención social, identifican la existencia de una doble intervención: una que respondería a los requerimientos de la ejecución del programa, y otra que responde a las acciones del vínculo y la construcción de confianza. Como plantea Alfredo Carballeda (2002, citado en Sánchez y Villarroel, 2017) implica la coexistencia de dimensiones de control y de emancipación en las acciones del programa.

\section{Adecuaciones del trabajo precario}

Pese a las tensiones generadas durante la ejecución en un marco de precariedad, los trabajadores y trabajadoras desarrollan ciertas adecuaciones, es decir, ciertas prácticas que les permiten modificar y acomodar algunos elementos para hacer más llevadera la situación precaria en la que se encuentran.

\section{La Colaboración}

Una de las prácticas características entre los y las trabajadoras del programa es la colaboración. Es posible identificar trabajo colaborativo allí donde éste no está prescrito. De este modo, los y las trabajadores se refieren a su trabajo como un espacio de confianza, de empatía, de aprendizaje, de cooperación el cual valoran y promueven. No sólo hay una práctica de compartir aquello que ha resultado, sino que también comparten sus dificultades y sus inquietudes respecto del trabajo que realizan. 
Por su parte, Francisca comenta en voz alta la siempre buena disposición de Luisa y lo buena compañera que ha sido. Karol agradece también la buena disposición de Luisa para enseñar "siempre aprendo de ti" le comenta. Luisa da la gracias a los nuevos y antiguos compañeros. Sigue el trabajo y Manuela se ofrece a recoger y ordenar los documentos, Josefina y Emilia comienza a ayudar en la nueva ordenación de las carpetas. (Entrada de diario de campo, mayo de 2016)

Las prácticas colaborativas permiten construir y compartir un aprendizaje, construyéndose estrategias situadas que hacen posible desarrollar una intervención desde las perspectivas que se señalan en las orientaciones técnicas. Como lo señala una de las trabajadoras, que además fue la última en incorporarse el equipo:

256.E2: =entonces en ese sentido sí pienso que aquí son súper colaboradores (.) no sé po cuando yo llegué igual:: la Karol que era otra tutora >altiro me dijo< cachó que yo andaba perdida y me agarro y me dijo varias cosas que me sirvieron harto (.hhh) y no sé po de repente hay chiquillos así podís trabajar e::sto y me dio hartos consejos, igual la Emilia:: entonces (.) sí >yo creo que en ese sentido los profesionales aquí no son pa na egoístas< súper solidarios, con el conocimiento, cosas que antes no sé $(x)$ en la universidad o en las otras pegas que había:: hecho la práctica ,para nada po (.) así la gente súper egoísta con el conocimiento po o sea no (x) es algo que:: no podís dejarlo para ti tenís que compartirlo porque en verdad va a mejorar todos los procesos (.hhh) de hecho de las primeras cosas que yo conversaba con la Emilia era la importancia de tener esa confianza y ese fiato con la tripleta (.hhh) que::: obviamente tenemos que estar todos remando pal mismo lado ,trabajando el mismo tema pa:: que las cosas resulten po (.) porque o si no imagínate tres ( $\mathrm{x}$ ) las tres haciendo >cada una por su lado< ¿a dónde llegamos?=

257. E1: $=[$ claro]

258. E2: [a ningu]na parte y al final los únicos que salen perjudicados son las familias po entonces está la importancia de compartir las experiencias de saber que el otro le cuesta má::s >o que tiene menos experiencia< en ese caso sí:: ,yo creo que (hhh) a veces digo en este mes he aprendido más cosas que en todo un semestre de la universidad=. (Josefina, entrevista personal, junio de 2016)

Las prácticas colaborativas incluso se realizan fuera del programa, una muestra es que, cuando el equipo debe trabajar con niños, niñas y familias de una ciudad aledaña, el equipo de un programa ejecutado por otra organización colaboradora, les facilita su infraestructura para que puedan desarrollar la intervención. 
Según Robert Castel (2003/2004) uno de los efectos del trabajo precario es la disminución de las regulaciones colectivas, de este modo es esperable que los trabajadores se vean desprendidos de sus pertenencias colectivas, afectándose el desarrollo de estrategias solidarias entre ellos. Esto, en el equipo investigado no se observa, dado que, pese al contexto precario, el equipo desarrolla prácticas colaborativas que se refuerzan en las crisis.

\section{LA HABILITACIÓN}

Resulta interesante la identificación de la habilitación como una estrategia de adecuación, en tanto permitiría valorar el paso por el programa, dada las condiciones de la precariedad.

La habilitación la comprendemos como aquella valoración del trabajo como una fuente de experiencia, una instancia para aprender e incorporar habilidades. Como señala un trabajador que, siendo trabajador social, desarrolla labores de educador dentro del programa:

146. E2: [claro] o sea igual es así:: >esta experiencia igual la tomo como experiencia profesional< siendo que no me estoy::: eh:: desarrollando como trabajador social lo que::: estudié:: pero aun así >es como una experiencia súper valiosa que sé que ya me va a abrir puertas para poder postular a cargos de trabajador social como tal<=. (Daniel, entrevista personal, junio de 2016)

Otra trabajadora, en las mismas condiciones, optó a este programa como primer trabajo, dado que significaba una oportunidad que no podía desaprovechar:

14. Como te dije cuando fui a la entrevista fui a una entrevista de trabajador social < pa un programa en CIUDAD (.hhh) y cuando yo tuve la entrevista allá en OFICINA me dijeron (.) "bueno puede ser pero >no tienes mucha experiencia< así que quizás puedas ser tutora" y yo le pregunté ¿qué es tutor-? $(\mathrm{x})$ bueno ¿qué hacen los $\uparrow$ tutores? Y me dijo "bueno >básicamente son estas las funcio::nes" y ahí me:: (x) me tincó me dijo ya sí >obviamente la remuneración es menos< pero::: igual es una oportunidad >y hay que aprovecharlas porque funo está recién egresado< y todo lo que entra::f sirve (.) entonces >yo dije ya::< sí igual sí >. (Josefina, entrevista personal, junio de 2016)

Incluso la llegada al trabajo es posible comprenderla desde la lógica de la habilitación. No necesariamente hay un interés de desarrollarse en las políticas de infancia, sino que trabajar para conocer cómo se desarrollan éstas y adquirir un aprendizaje. Así, la experiencia laboral está asociada a tener más oportunidades laborales, es decir, adquiere relevancia en tanto posibilidad futura. 
334. E2: =>entonces aproveché la experiencia aparte que igual estaba buscando $\downarrow$ trabajo $<$ y en esos casos a vece::s uno:: acepta lo que viene=

335. E1: =claro o sea estabas [buscando]

336. E2: [yo dije] esta es una organización que es conocida:: relevante $>$ trabajar en - CIUDAD< ya:: perfecto entonces he estado en esa >desarrollando experiencia< más que como aprendizaje (.hhh) >o sea hay aprendizaje $\downarrow$ constante $>$ pero más que una proyección en el área $<$ un aprendizaje= 337. $\mathrm{E} 1:=\mathrm{m}::=$

338. E2: =porque igual a mí:: me interesa:: desarrollar como otras áreas que son:: (x) que no son propias del trabajo social ¿cachai? (.hhh) son áreas má:: $\mathrm{s}>$ más ligadas a la ciencia social<=

339. $\mathrm{E} 1:=\mathrm{mhm}=$

340. E2: =>así que igual< yo ando buscando experiencia >pa poder tener (.) más oportunidades laborales< más currículum (.hhh) bueno poder aprender también y poder desarrollar una buena labor en los contextos donde esté (.hhh) pero así también (.) poder ir escalando hasta alcanzar mis objetivos que no están ligados como con áreas ((golpea la mesa con los dedos)) específicas del trabajo social=

341. E1: =mhm ya >entonces como que $<$ lo tomas como una experiencia que no solamente es profesional sino también es como vital::=

342. E2: =sí po [sí::]

343. E1: [y::] desde ahí armai como una proyección::=

344. E2: =sí:: totalmente cuando yo llegué igual >mi pensamiento es como voy a tratar no sé< de estar como:: dos a::ños en este $\downarrow$ trabajo=

345. $\mathrm{E} 1:=\mathrm{m}:: \mathrm{mhm}=$

346. E2: $=0$ en cualquier otro trabajo >a menos que sea como los que::< esté buscando a futuro es cosa de ir:: desarrollando experiencia >aparte que igual soy joven::< (.hhh) >y ya estoy trabajando::< entonces es como (.) es momento de aprovechar e ir adquiriendo distintas experiencias laborales (.hhh) e ir elaborando también::: un discurso profesional=. (Daniel, entrevista personal, marzo de 2016)

Esta consideración del trabajo como habilitación tiene asidero dentro de la organización, puesto que dentro de la misma se da una promoción de los trabajadores que se desempeñan en los diferentes programas. De esta manera, cuando un programa se cierra, van pasando a otro y van desarrollando funciones más especializadas, incluso esto ocurre dentro de un mismo programa.

No obstante, algunos trabajadores establecen críticas de lo que implica que este trabajo sea desarrollado por profesionales que estén buscando aprender y habilitarse. Una de las principales observaciones es que se está en permanente construcción, no llegándose a una ejecución fluida con equipos afiatados. Al respecto una trabajadora señala: 
276. E2: =porque también varios amigos han:: les ha pasado que no lo sostienen ¿cachai? $\mathrm{O}$ sea se van con las historias pegadas en los oídos (.hhh) y no:: y no pueden más y lo repiten en su casa:: ¿cachai? $Y$ yo en ese sentido siento que $(\mathrm{x})$ que por algo:: $\uparrow$ funciono entonces tengo que entregar eso ,pa qué me voy a ir a:: $(\mathrm{x})$ a algo como:: si $(\mathrm{x})$ si tengo la posibilidad de hacerlo si tengo los recursos >por otro lado siento que soy una buena profesional< (.hhh) me carga el tema de las políticas públicas que al final terminan quedándose solamente los nuevos porque no tienen otra pega=

277. E1: =m: sí-=

278. E2: =y una vez que ya::: eh::=

279. E1: =tenís [experiencia]

280. E2: [tienen ex]periencia tran- $(x)$ se saltan a otro lado que también es súper válido por sus expectativas laborales (.hhh) eh:: pero $(x)$ pero en mi caso ahora no sé cómo será con dos hijos el escenario cambia ${ }^{\circ}$ son distintas $^{\circ}$ responsabilidades eh:: ahí empiezan a velar otras co::sas la:: (x) la ideología pierde un poco:: el peso=. (Francisca, entrevista personal, mayo de 2016)

Esta habilitación responde a las nociones de empleabilidad, toda vez que el aprendizaje y la incorporación de habilidades y competencias, permite a los trabajadores y trabajadoras acceder a mejores trabajos en el futuro, o al menos les permite considerar esto como una posibilidad.

La habilitación conduciría a acumular lo que Michel Foucault (2004/2007) comprende como “capital humano", es decir, un conjunto de competencias, aptitudes, talentos de los que el trabajador puede disponer para vivir y trabajar.

Este capital humano sería llevado por el trabajador o trabajadora de una relación laboral a otra, constituyéndose de este modo en un "trabajador portafolio" (Handy, 1996, citado en Sisto, 2009). El o la trabajadora de la política social puede migrar a otro programa, en otra organización, llevando lo aprendido - su capital humano- y agregando más capital para ofrecer en una futura relación laboral.

\section{CONCLUSIÓN}

Las prácticas de los trabajadores y trabajadoras del programa social de infancia nos convocan a una reflexión en torno a cómo las condiciones de ejecución favorecen determinadas construcciones de sujeto.

A partir de un análisis foucaultiano, el trabajador, en tanto sujeto situado en condiciones precarias, se constituye como un homo œconomicus, es decir, un empresario de sí mismo que ve en la posibilidad de trabajar - aun en condiciones precarias- una opción de incrementar su capital humano, que es la fuente 
de sus ingresos (Foucault, 2004/2007). Paradojalmente, para el trabajador y la trabajadora el capital humano no es valioso en tanto promueve un ejercicio en el presente, sino que en tanto asegura un potencial empleo en el futuro.

De ese modo, como señala Luca Paltrinieri (2013), tiene origen la condición esquizofrénica del sujeto neoliberal, en tanto se evalúa en el presente respecto de lo que podrá ser en el futuro. Esta condición esquizofrénica es lo que hace su vida precaria y gobernable, evalúa el conjunto de sus habilidades en el presente pero esa evaluación concierne una futura realización de sus competencias, que nunca llega.

Podríamos reflexionar en torno al desarrollo de los programas sociales de infancia -que son parte de la acción social del Estado neoliberal-como verdaderas tecnologías políticas del individuo del neoliberalismo.

Así, el neoliberalismo como un arte de gobernar estructura -estructurando el medio- a un sujeto que en libertad decide respecto de su trayectoria laboral, desde la aparente aceptación de las condiciones precarias. Como señala Foucault (1982), el gobierno puede ser sólo ejercido en sujetos libres, en los cuales una variedad de formas de comportarse puedan ser posibles.

El trabajador y la trabajadora se encuentran impelidos a fortalecer su capital humano, en ausencia de teorías sustantivas que lo soporten en su accionar cotidiano.

Por su parte, la organización es educadora -es escuela - y al mismo tiempo utiliza las habilidades y competencias de este trabajador, sabiendo que su paso por el programa es temporal.

El trabajador y la trabajadora de la política social de infancia es un sujeto versátil, todo terreno, que puede entrar en el vínculo y desvincularse con igual rapidez si queda fuera de la relación laboral, y que puede responder a múltiples demandas de manera simultánea.

De este modo se constituye como un sujeto paradojal, desbordado por la orientación contradictoria de trabajar desde el vínculo en un marco que hace que éste sea inestable y frágil, dada la precariedad y sus consecuencias. Este sujeto paradojal se sobrepasa a si mismo toda vez que sostiene esta contradicción, comprometiendo en ello sus esfuerzos y concibiendo esta experiencia como una oportunidad de aprendizaje: la habilitación. Desde ahí que la constante incertidumbre que lo caracteriza, la ansiedad y potencialmente el desgaste laboral (De Gaulejac y Hanique, 2015) son incorporadas en los relatos de sí como una circunstancia, como una etapa, tras la cual llegará un mejor empleo. 
La habilitación aparece como un recurso simbólico clave en la constitución del sujeto trabajador de la política social de infancia. Se despliega como un soporte subjetivo para convivir con la contradicción, a la vez que permite justificar su permanencia en un trabajo precario sin comprometer su valoración de sí mismo como profesional. En esta adecuación se expresa la dominación simbólica de las políticas públicas, tal como plantea Vincent Dubois (2015/2020).

Sin embargo, este proceso no está exento de resistencias, entre las cuales podemos encontrar fundamentalmente la cooperación como práctica de colectivización del conocimiento, ayuda mutua y trabajo colaborativo (Lorenzini, 2018). Estas prácticas colectivas dan origen a particulares formas de trabajar, situadas y creativas, que permiten desarrollar la labor en el programa.

Las prácticas de cooperación que despliegan las y los trabajadores les permiten llevar a cabo su labor, cumplir el objetivo del programa. Pareciera que, sin las estrategias de ayuda mutua, no se podría desarrollar el trabajo en los términos en los que se plantea en las orientaciones estatales.

De este modo, podemos ver cómo el carácter neoliberal de las políticas de infancia, que supone un determinado funcionamiento, no es un objeto dado, sino que está sujeto a acomodaciones. En la política social de infancia, el imperativo neoliberal se construye y re-construye, facilitado por ciertos puntos de apoyo tales como las licitaciones y la primacía de los indicadores cuantitativos y su registro. No obstante, se sostiene en un fundamento administrativo en crisis, hiperactivo en casos de alta connotación pública y en constante reforma.

Desde ahí, uno de los alcances relevantes del presente estudio es la profundización en un programa social de infancia y la posibilidad de aproximarse a la ejecución de la política social de infancia cuyo enfoque de derechos se ve seriamente comprometido dada las condiciones de precariedad en las que se desarrollan las prácticas de su ejecución.

Las limitaciones de este estudio radican en que se trata de un análisis de un solo programa que es parte de la política social de infancia, viéndose limitado el alcance de los resultados y análisis que aquí se presentan.

Por otra parte, una relevante pregunta para futuros estudios es aquella por las implicancias que esto tiene para los niños, niñas y familias que participan de los programas. Si bien se prescribe el trabajo desde un enfoque de derechos, se concibe al niño como un usuario, un beneficiario, que no es expulsado de la intervención, pero que debe soportar el cambio de los profesionales con los que se vincula, viéndose transgredido desde esta inestabilidad (Pavez et al., 2019). 
El estudio nos permite aproximarnos al campo de la política social de infancia y a la batalla que allí se estaría articulando, una pelea por la sujeción a una construcción neoliberal de sujetos emprendedores y consumidores de políticas, versus la construcción de una ciudadanía que se encuentra en un espacio con sentido, con un horizonte de transformación de las condiciones de vida, en la construcción de una sociedad que compartimos y en la que nos vinculamos.

Esto resulta de interés no solo para los propios trabajadores y trabajadoras, sino que también para las organizaciones ejecutoras de estos programas y para la sociedad civil comprometida en la construcción de una sociedad en la que niños y niñas vivan en un marco de respeto a sus derechos.

En esta sociedad compartida, sin duda que las instituciones formadoras tienen mucho que aportar, siendo también de interés para ellas este estudio. Cabe la pregunta por la formación ética de los profesionales, dado que este contexto de ejecución supone tensiones que interpelan las visiones del otro y de la sociedad (Winkler y Reyes, 2017). De este modo, la ética -en constante construcción- es un elemento que está presente de manera transversal y que se encuentra tensionado en la labor que desarrollan los trabajadores y trabajadoras de los programas sociales de infancia en Chile.

\section{REFERENCIAS}

Álvarez, Isabel \& Sáenz, Stella. (2014). Las políticas sociales en América Latina y el Caribe: Perspectivas y desafíos. En Adrián Bonilla, Isabel Álvarez \& Stella Sáenz (Eds.), Políticas sociales en América Latina y el Caribe: Escenarios contemporáneos, inversiones y necesidades (pp. 15-23). FLACSO.

Ascorra, Paula (2009). El gerenciamiento de los estados de ánimo: Estudio de caso en una organización chilena. Irice, 20, 21-32.

Barros Aguirre, Daniel (2006). Pobreza y políticas públicas en Nicaragua década de los noventa. Tesis de Maestría sin publicar, FLACSO.

Bezes, Philippe; Demazière, Didier; Le Bianic, Thomas; Paradeise, Catherine; Normand, Romuald; Benamouzigb, Daniel; Pierru, Frederic \& Evetts, Julia (2012). New public management and professionals in the public sector: What new patterns beyond opposition?. Sociologie du Travail, 54(1), 1-52.

https://doi.org/10.1016/j.soctra.2012.07.001

Bessin, Marc. (2014). Présences sociales: une approche phénoménologique des temporalités sexuées du care. Temporalités, 20, 1-15.

https://doi.org/10.4000/temporalites.2944

Castel, Robert (2003/2004). La inseguridad social ¿Qué es estar protegido? Manantial.

Coutant, Isabelle \& Eideliman, Jean-Sébastien (2013). The moral economy of contemporary working-class adolescence: managing symbolic capital in a French public Adolescent Centre. The British Journal of Sociology, 64(2), 248-266.

https: / / doi.org/10.1111/1468-4446.12016 
Dávila, Oscar (1998). Estado y Políticas Sociales: Del estado protector al estado subsidiario. Última Década, 9, 105-120.

De La Maza, Francisca (2012). Etnografía de las prácticas sociales y negociaciones en la política indígena actual, Araucanía-Chile. Revista Austral de Ciencias Sociales, 22, 85-100. https://doi.org/10.4206/rev.austral.cienc.soc.2012.n22-05

De Gaulejac, Vincent \& Hanique, Fabienne (2015). Le Capitalisme paradoxant: Un système qui rend fou. Éditions du Seuil.

Dubois, Vincent (2012). Ethographier l'action publique: Les transformations de l'État social au prisme de l'enquête de terrain. Gouvernement \& Action Publique, 1, 87105. https://doi.org/10.3917/gap.121.0083

Dubois, Vincent (2015/2020). Sujetos en la burocracia: Relación administrativa y tratamiento de la pobreza. UAH Ediciones

Fardella, Carla; Sisto, Vicente; Morales, Karol; Rivera, Guillermo \& Soto, Rodrigo (2016). Identidades Laborales y Ética del Trabajo Público en Tiempos de Rendición de Cuentas. Psykhe, 25(2). http://dx.doi.org/10.7764/psykhe.25.2.789

Fassin, Didier (2010). El irresistible ascenso del derecho a la vida. Revista de Antropología Social, 19, 191-204.

Fine, Michelle (1994). Working the hyphens: Reinventing self and other in qualitative research. En Norman K. Denzin \& Yvonna S. Lincoln (Eds.), Handbook of qualitative research (pp. 70-82). Sage Publications, Inc.

Flick, Uwe; Von Kardorff, Ernst \& Steinke, Ines (2004). A companion to qualitative research. Sage Publications.

Foucault, Michel (1982). The Subject and Power. Critical Inquiry, 8(4), 777-795. https://doi.org/10.1086/448181

Foucault, Michel (2004/2007). Nacimiento de la biopolítica. Fondo de Cultura Económica.

Geertz, Clifford. (2005). La interpretación de las culturas. Gedisa.

Haz, Ana María; Bilbao, M. Ángeles \& Canales, Lilian (2012). Gestión de los Equipos. En Teresa Matus (Eds), Desarrollo de un modelo de gestión de calidad de programas sociales. (pp. 213-252). Librosdementira Ltda.

Hood, Cristopher (1991). A public management for all seasons? Public Administration, 69(1), 3-19. http://dx.doi.org/10.1111/j.1467-9299.1991.tb00779.x

Íniguez, Lupicinio (1999). Investigación y evaluación cualitativa: bases teóricas y conceptuales. Atención Primaria, 23(8), 496-502.

Jeffrey, Bob \& Troman, Geoff, (2004). Time for ethnography. British Educational Research Journal, 30(4), 535-548. https://doi.org/10.1080/0141192042000237220

Ley N $N^{\circ} 20.032$. Diario Oficial de la República de Chile, Santiago, Chile, 25 de julio de 2005.

Lipsky, Michel (1980). Street-level bureaucracy. Russell Sage Foundation.

Lorenzini, Daniele. (2018). Governmentality, subjectivity, and the neoliberal form of life. Journal for Cultural Research, 22, 1-14. https://doi.org/10.1080/14797585.2018.1461357 
Núñez, Carmen Gloria; Solís, Camila \& Soto, Rodrigo (2014). ¿Qué sucede en las comunidades cuando se cierra la escuela rural? Un análisis psicosocial de la política de cierre de las escuelas rurales en Chile. Universitas Psychologica, 13(2), 615-625. https://doi.org/10.11144/javeriana.upsy13-2.qscc

Paltrinieri, Luca (2013). Quantifier la qualité. Le capital « humain » entre économie, démographie et éducation. Raisons Politiques, 52, 89-107. https://doi.org/10.3917/rai.052.0089

Pavez, Javiera; Herrera, Yesica; Molina, Javier; Ortúzar, Harry \& Zamora, Carlos (2019). El permanente conflicto entre Estado e Instituciones colaboradoras a partir de su marco normativo. Athenea Digital, 19(1), e2201.

https: / / doi.org/10.5565/rev/athenea.2201

Ramírez, María Fernanda (2009). Las reformas del Estado y la administración pública en América Latina y los intentos de aplicación del New Public Management. Estudios Políticos, 34, 115-141.

Sánchez, Mauricio \& Villarroel, Rosa (2017). Tensiones en la Intervención Social: (des)encuentros en la relación Estado-ONG. Estudio de caso sobre ONG que opera la política social de infancia. Revista de Trabajo Social, 91, 3-16.

Sharma, Aradhana \& Gupta, Akhil (Eds.) (2006). The anthropology of the state: A reader. Blackwell Publishing.

Silva, Carlos \& Burgos, César (2011). Tiempo Mínimo-Conocimiento Suficiente: la cuasietnografía sociotécnica en psicología social. Psicoperspectivas, 10(2), 87-108. https://dx.doi.org/10.5027/psicoperspectivas-Vol10-Issue2-fulltext-146

Sisto, Vicente (2009). Cambios en el trabajo, Identidad e Inclusión Social en Chile: desafíos para la investigación. Revista UNIVERSUM, 2(24), 192-216. http://dx.doi.org/10.4067/S0718-23762009000200011

Street, Susan (2003). Representación y Reflexividad en la (auto) etnografía crítica: ¿Voces o Diálogos? Nómadas, 18, 72-79.

Torres, Osvaldo (2009). Niñez, políticas públicas y sociedad civil. Revista MAD, 3, 17 45. https://doi.org/10.5354/0718-0527.2008.31052

Wanderley, Fernanda (2009). Prácticas estatales y el ejercicio de la ciudadanía: encuentros de la población con la burocracia en Bolivia. Íconos, 34, 67-79. https://doi.org/10.17141/iconos.34.2009.334

Winkler, María Inés \& Reyes, Maria Isabel. (2017). La aceptación de la diversidad en cuestión: Experiencias enriquecedoras y adversas en la formación y la práctica profesional temprana en psicología. Acta Bioethica, 23(1), 99-108. https: / / doi.org/10.4067/S1726-569X2017000100099 


\section{JaViera Beatriz PaVez Mena}

Profesora asociada en el ámbito de la Psicología Comunitaria, en la Escuela de Psicología de la Pontificia Universidad Católica de Valparaíso. Desarrolla investigación en el campo de la política social, especialmente la política social de infancia. javiera.pavez@pucv.cl

\section{FORMATO DE CITACIÓN}

Pavez Mena, Javiera Beatriz (2021). Tensiones y adecuaciones de los trabajadores en el marco de la ejecución de la política social chilena. Quaderns de Psicologia, 23(2), e1610. https://doi.org/10.5565/rev/qpsicologia.1610

HISTORIA EDITORIAL

Recibido: 21-01-2020

$1^{\text {a }}$ revisión: $30-06-2020$

Aceptado: $16-10-2020$

Publicado: 31-08-2021 